\title{
A Data Mining Approach for Jet Grouting Uniaxial Compressive Strength Prediction
}

\author{
Joaquim Tinoco; António Gomes Correia \\ Dep. of Civil Engineering/C-TAC \\ University of Minho \\ Guimarães, Portugal \\ jabtinoco@civil.uminho.pt; agc@,civil.uminho.pt
}

\author{
Paulo Cortez \\ Dep. of Information Systems/Algoritmi R\&D Centre \\ University of Minho \\ Guimarães, Portugal \\ pcortez@dsi.uminho.pt
}

\begin{abstract}
Jet Grouting (JG) is a Geotechnical Engineering technique that is characterized by a great versatility, being the best solution for several soil treatment improvement problems. However, JG lacks design rules and quality control. As the result, the main JG works are planned from empirical rules that are often too conservative. The development of rational models to simulate the effect of the different parameters involved in the JG process is of primary importance in order to satisfy the binomial safety-economy that is required in any engineering project. In this work, three data mining models, i.e. Artificial Neural Networks (ANN), Support Vector Machines (SVM) and Functional Networks (FN), were adapted to predict the Uniaxial Compressive Strength (UCS) of JG laboratory formulations. A comparative study was held, by using a dataset used that was obtained from several studies previously accomplished in University of Minho. We show that the novel data-driven models are able to learn with high accuracy the complex relationships between the UCS of JG laboratory formulations and its contributing factors.
\end{abstract}

Keywords: Ground improvement; Jet grouting; Uniaxial compressive strength; Artificial Neural Netwoks; Data Mining.

\section{INTRODUCTION}

Currently, there are several techniques for soil improvement where the Jet Grouting (JG) technology is highlighted. This technology is sharply growing and it is characterized by a great versatility in a variety of application soil types and treatment geometries and it has been applied to several Geotechnical Engineering tasks [1][2][3]. The JG technology consists in injecting a high speed grouting of water-cement mixtures and/or other (e.g. air, water) into the subsoil. The fluids are injected through small-diameter nozzles placed on a rod, which is continually rotated to slowly remove soil. Currently adopted JG methods can be classified according to the number of fluids injected into the subsoil: water-cement grout - single-fluid system; air + grout - double-fluid system; and water + air + grout - triple-fluid system.

This paper will focus the JG initial stage, where a set of laboratory formulations, which are function of the soil type to be treated and the design properties, are used to set the soil-cement mixture that will be used in the construction works. In particular, this study allows the definition of the grout water/cement ratio, the amount of cement for cubical meter of treated soil and the cement type, needed to satisfy the design and economical requirements. The remaining parameters that control the final characteristics of the JG elements (e.g. the speed of withdrawal and rotation of the rods), will be evaluated with the execution of test columns, given the difficulty to simulate such parameters in laboratory. After the establishment of all parameters, the construction works of the columns are initiated. Next, after a period of time, samples are extracted and tested to evaluate the treatment quality and eventually proceed to adjustments of those same parameters. This control procedure, together with the use of predictions of the mechanical behaviour of the materials, aims at the estimation of the physical and mechanical properties of the treated material over time. Hence, it aids to perform eventual adjustments in the works. However, at the design stage of JG, there are still uncertainties because there are no reliable methods that allow the prediction of the diameters and the mechanical properties of the soil-cement elements [4][5]. Thus, given the high potential that the JG technology presents, there is need to develop more rigorous and accurate models of design. This will allow a reduction of field tests, optimizing all the constructive process and obtaining a higher technical and economical efficiency.

To achieve this goal, we propose the application of data mining techniques, namely Artificial Neural Networks (ANN), Support Vector Machines (SVM) and Functional Networks (FN). We also adapted the EC2 analytical model [6], often used to predict the Uniaxial Compressive Strength (UCS) of concrete, to JG material. ANN have become increasingly used since the introduction of the backpropagation algorithm [7]. More recently, SVM have also been proposed [8]. Due to their higher flexibility and nonlinear learning capabilities, these methods are gaining an attention within the data mining (DM) field, often attaining high predictive performances [9]. Indeed, SVM, ANN and FN have been successful used to solve a wide range of problems in statistics [10], science [11], pattern recognition [12], structural civil engineering [13], and another civil engineering domains $[14][15][16]$. Yet, within our 
knowledge, these techniques have not been applied to JG data, namely to prediction of UCS, which is a complex geotechnical engineering problem due to the heterogeneous nature of the soil and the large number of the parameters involved.

\section{MATERIALS AND METHODS}

\subsection{Jet Grouting Data}

The dataset includes 175 results of UCS tests, derived from $35 \mathrm{JG}$ laboratory formulations and 9 input parameters, which are referred as the more influent parameters in UCS prediction [17][18]:

- Water/Cement ratio - W/C;

- Coefficient related to the cement type ( 0.25 for CEM IV 32.5R and 0.20 for CEM I 42.5R and CEM I 42.5R) - s;

- $\quad$ Age of the mixture - $\mathbf{t}$

- The relation between the mixture porosity and volumetric content of cement - n/(Civ) ${ }^{\mathbf{d}}$;

- $\quad$ Percentage of cement of the mixture - $\% \mathbf{C}$;

- Percentage of sand - \%Sand;

- $\quad$ Percentage of silt - \%Silt;

- Percentage of clay - \%Clay;

- Percentage of organic matter - \%OM.

The main statistics of the numerical parameters used are presented in Table I. The dataset compiled resulted of a study accomplishment in University of Minho with the goal of analyzing the influence of several parameters in UCS of $\mathrm{JG}$ materials. The soils used in the preparation of $\mathrm{JG}$ laboratory formulations come from seven field works. The geotechnical soil properties were evaluated using laboratory tests and the respective soil classifications are presented in Table II. While all of the soils were classified as inorganic fine soil, they have different percentages of sand, silt, clay and organic matter. The cement types used were CEM I 42.5R, CEM II 42.5R and CEM IV/A 32.5R. Fig. 1 plots the histogram of the target variable, showing a log-normal distribution shape.

TABLE I. SYNOPSIS OF THE NUMERICAL PARAMETERS (INPUTS)

\begin{tabular}{|c|c|c|c|c|c|}
\hline Soil & Parameter & Minimum & Maximum & Mean & $\begin{array}{l}\text { Standard } \\
\text { deviation }\end{array}$ \\
\hline \multirow{9}{*}{ Clay } & $\mathrm{W} / \mathrm{C}$ & 0.68 & 1.12 & 0.88 & 0.16 \\
\hline & s & 0.20 & 0.25 & 0.21 & 0.02 \\
\hline & t (days) & 3.00 & 56.00 & 22.00 & 19.00 \\
\hline & $\mathrm{n} /(\mathrm{Civ})^{\mathrm{d}}$ & 48.83 & 74.26 & 62.59 & 7.26 \\
\hline & $\% \mathrm{C}$ & 24.19 & 73.98 & 47.44 & 0.15 \\
\hline & $\%$ Sand & 0.00 & 39.00 & 13.47 & 11.54 \\
\hline & $\%$ Silt & 33.00 & 57.00 & 50.49 & 5.49 \\
\hline & $\%$ Clay & 22.50 & 45.00 & 35.87 & 7.74 \\
\hline & $\% \mathrm{OM}$ & 0.40 & 8.30 & 2.71 & 1.81 \\
\hline
\end{tabular}

Nowadays, there are several models to estimate the UCS of concrete over time [6][19]. However the EC2 analytical is the most popular. Thus, despite this model is defined for concrete material, it was tested for the JG laboratory formulation, due to the similarity of the materials. According to this model, the evolution of the UCS over time is given by the following expression:

$$
q(t)=e^{\left(s \cdot\left[1-\left(\frac{28}{t}\right)^{1 / 2}\right]\right)} \cdot f_{c m}
$$

The adopted values for the coefficients in (1) were: $q$ strength at age $t(\mathrm{MPa}) ; f_{c m}-28$ day strength for each studied formulation (MPa); $s=0.2$ to cement CEM I 42.5R and CEM II 42,5R and $s=0.25$ to cement CEM IV/A (V) 32.5R; and $t$ - age of the respective formulation in days.

TABLE II. SUMMARY OF THE SOIL ClassificAtions

\begin{tabular}{llrrrrr}
\hline Soil & Classification & $\begin{array}{r}\% \\
\text { Sand }\end{array}$ & $\begin{array}{r}\text { \% } \\
\text { Silt }\end{array}$ & $\begin{array}{r}\% \\
\text { Clay }\end{array}$ & $\begin{array}{r}\% \\
\text { OM }\end{array}$ & $\begin{array}{r}\mathbf{N}^{\mathbf{0}} \text { of } \\
\text { samples } \\
\text { prepared }\end{array}$ \\
\hline A & Lean clay (CL) & 39.0 & 33.0 & 27.0 & 8.3 & 10 \\
B & Organic lean clay (OL) & 6.0 & 57.0 & 37.0 & 1.8 & 5 \\
\hline C & Fat clay (CH) & 7.0 & 53.0 & 40.0 & 3.2 & 85 \\
D & Silty clay (CL-ML) & 25.0 & 52.5 & 22.5 & 0.4 & 20 \\
E & Lean clay (CL) & 0.0 & 55.0 & 45.0 & 3.9 & 15 \\
F & Silty clay (CL-ML) & 32.5 & 43.5 & 24.0 & 1.2 & 20 \\
G & Lean clay (CL) & 10.5 & 48.5 & 41.0 & 1.0 & 20 \\
\hline
\end{tabular}

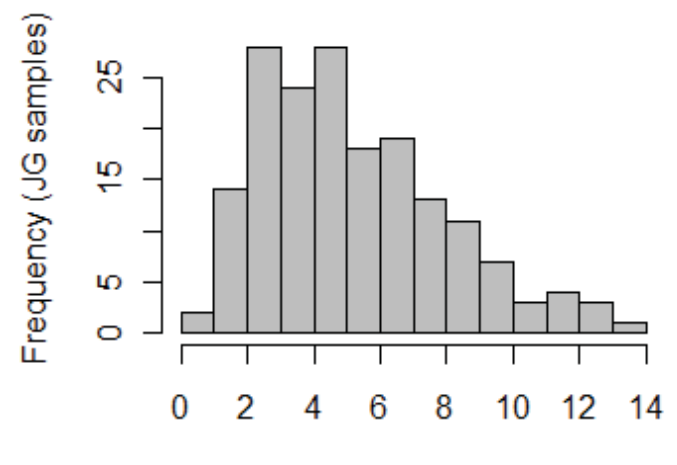

Uniaxial Compressive Strength (MPa)

Figure 1. The histogram for the UCS of the JG laboratory formulations

\subsection{Predictive Models}

We apply three different DM techniques to estimate the UCS of JG material over time, which is a regression task. We adapted ANN, SVM and FN. Despite of high complexity of first two models, it is still possible to extract knowledge in terms of input variable importance [20].

ANN mimics some basic aspects of brain functions [21], which processes information by means of interaction among several neurons [22]. We will adopt the most popular ANN model, the multilayer perceptron, which contains only feedfoward connections, with one hidden layer with $\mathrm{H}$ processing units. A network with $\mathrm{H}=0$ is equivalent to the multiple regression model. By increasing $\mathrm{H}$, more complex mappings can be performed, yet an excess value of $\mathrm{H}$ will overfit the data, leading to a loss of generality. To overcome this difficult, a grid search $\{2,4, \ldots, 10\}$ was used to choose best $H$ value [9]. We adopted an internal 5-fold cross validation over the training set and then selected the network with the lowest validation error. Next, this ANN was 
retrained with all training data. The general model of the $\mathrm{ANN}$ is:

$$
\hat{y}=W_{o, O}+\sum_{j=I+1}^{o-1} f\left(\sum_{i=1}^{I} X_{i} \cdot W_{j, i}+W_{j, O}\right) \cdot W_{o, i}
$$

where $W j, i$ represents the weight of the connection from neuron $j$ to the unit $i$ (if $j=0$, then it is a bias connection), $o$ corresponds to an output unit, $f$ is a logistic function $\left(\frac{1}{1+e^{-x}}\right)$, and $I$ is the number of input neurons.

The SVM was initially proposed for classification tasks (i.e., to model a discrete labeled output). After the introduction of the $\varepsilon$-insensitive loss function, it was possible to apply SVM to regression tasks [23]. SVM has theoretical advantages over ANN, such as the absence of local minima in the learning phase, i.e., the model always converges to the optimal solution. The main idea of the SVM is to transform the input data into a high-dimensional feature space by using a nonlinear mapping $\phi$. Then, the SVM finds the best hyperplane within the feature space. The transformation depends on the kernel function adopted. The Gaussian kernel is the most popular one, presenting less parameters than other kernels, and will be adopted in this work:

$$
k\left(x, x^{\prime}\right)=e^{\left(-\gamma \times\left\|x-x^{\prime}\right\|^{2}\right)}, \quad \gamma>0
$$

Under this setup, performance of the regression is affected by three parameters: $\gamma$ - the parameter of the kernel, $C$ - a penalty parameter, and $\varepsilon$ - the width of a $\varepsilon$-insensitive zone. To reduce the search space, the first two values will be set using the heuristics of [24]: $C=3$ (for a standardized output) and $\varepsilon=\hat{\sigma} / \sqrt{N}$, where $\hat{\sigma}=1.5 / N \times \sum_{i=1}^{N}\left(y_{i}-\widehat{y}_{l}\right)^{2}$ and $\widehat{y}_{l}$ is the value predicted by a 3-nearest neighbor algorithm. To optimize the Kernel parameter $\gamma$, we adopted a grid search of $\left\{2^{-15}, 2^{-13}, \ldots, 2^{3}\right\}$, which works as explained for ANN.

FN are a general framework useful for solving a wide range of problems (e.g. statistics and engineering applications) and it has been successfully used in solving both prediction and classification problems [25][26][27]. In these types of networks, the functions of the neurons can be multivariate, multi-argument and it is also possible to use different learnable functions, instead of fixed functions. Moreover, there is no need to associate weights to connections between nodes, since the learning is achieved by the neural functions. It should be noted that these functions are not arbitrary but subject to strong constraints to satisfy the compatibility conditions imposed by the existence of multiple links going from the last input layer to the same output units. When compared with ANNs, there are some advantages [28]. Unlike ANN, FN can reproduce certain physical characteristics that lead to the corresponding network in a natural way. However, such reproduction only takes place if we use an expression with a physical meaning inside the function database. Also, the estimation of the network parameters can be obtained by resolving a linear system of equations, which returns a fast and unique solution, i.e. the global minimum is always achieved.

FN and ANN have a similar structure, but they also have important differences. While in the functional network the selection of the initial topology is normally based on the properties of the problem at hand, in ANN, several topologies are considered and one is chosen using an optimal criterion. The initial topology in functional networks can be further simplified using functional equations and its neural functions can be multidimensional and set during the learning phase. FN incorporates different neural functions, normally functions from a given family, such as polynomial or exponential, and they are not restricted to be a linear combination of inputs. Furthermore, neurons outputs can be connected, which is not the case of standard ANN.

The structure of a FN consists in: a layer of input storing units; a layer of output storing units; one or several layers of processing units, which evaluate a set of input values, coming from the previous layer and delivers a set of output values to the next layer; none, one or several layers of intermediate storing units, and a set directed links, that connect units in the input or intermediate layers to neuron units, and neuron units to intermediate or output units.

When working with FN, several steps are necessary to be set. The first step is to define the initial topology of the network, based on problem in hand. Next, the architecture using functional equations and the equivalence concept needs to be initialized, and then checked the uniqueness condition of the desired architecture. Third, using the available data, the learning procedure (i.e. training algorithm) is realized by considering the combinations of linear independent functions, $\psi=\left\{\psi_{s 1}, \ldots, \psi_{s m_{s}}\right\}$, for all $s$ to approximate the neuron functions, that is:

$$
g_{s}(x)=\sum_{i=1}^{m_{s}} \alpha_{s i} \cdot \psi_{s i}(x) \quad \text { for all } s
$$

where the coefficients $\alpha_{i}$ are the parameters in functional networks. The most common linearly independent functions are: $\psi=\left\{1, X, \ldots, X^{m}\right\} ; \psi=\left\{1, e^{X}, e^{-X}, \ldots, e^{m X}, e^{-m X}\right\}$ or $\psi=\left\{1, \cos (X), \ldots, \cos ^{l}(X), \sin ^{l}(X)\right\}, m=2 l$, where $m$ is the number of elements in the combination of sets of linearly independent function.

To learn the parameters in (4), we can use one of the known optimizations techniques, such as last squares estimation, conjugate gradient, iterative last squares, minimax or maximum like likelihood estimation. The last step in implementation process is to select the best model and validate it.

In this work we use the FN to solve the following generic expression:

$$
\hat{y}=\beta_{0} \cdot \prod_{i=1}^{n} x_{i}{ }^{\alpha_{i}}
$$

where, $\left\{x_{1}, \ldots, x_{i}\right\}$ are the input parameters, $\left\{\beta_{0}, \alpha_{1} \ldots, \alpha_{i}\right\}$ are the coefficients to be adjusted.

To learn the coefficients in (5) the following minimization problem was used:

$$
\text { Minimize } Q=\sum_{S=1}^{S} \delta_{S}{ }^{2}=\sum_{S=1}^{S}\left(y_{S}-\beta_{0} \cdot \prod_{i=1}^{I} x_{i}{ }^{\alpha_{i}}\right)^{2}
$$


The ANN and SVM models were training using the R statistical tool [29] and the RMiner library [30], which facilitate the application of DM techniques in $\mathrm{R}$ and the formulation and resolution of the FN implemented in the free version of the GAMS [31].

\subsection{Evaluation}

In order to evaluate the performance of the prediction models and choose the best model, we consider three commonly used evaluative measures: Mean Absolute Error (MAD), Root Mean Absolute Error (RMSE) and Coefficient of determination $\left(\mathrm{R}^{2}\right)$ :

$$
\begin{gathered}
M A D=\frac{\sum_{i=1}^{N}|y-\hat{y}|}{N} \\
R M S E=\sqrt{\frac{\sum_{i=1}^{N}(y-\hat{y})^{2}}{N}} \\
R^{2}=\left(\frac{\sum_{i=1}^{N}(y-\bar{y}) \times(\hat{y}-\bar{y})}{\sqrt{\sum_{i=1}^{N}(y-\bar{y})^{2} \times \sum_{i=1}^{N}(\hat{y}-\overline{\hat{y}})^{2}}}\right)^{2}
\end{gathered}
$$

where $y$ denotes the desired value, $\hat{y}$ the predicted value, $\bar{y}$ and $\overline{\hat{y}}$ represent the mean of these variables. Lower values of MAD and RMSE correspond to a higher predictive capacity, while the $\mathrm{R}^{2}$ should be close to the unit value. If it is acceptable to commit a few extreme errors, then the MAD is the most adequate measure; else the RMSE measure should be used.

After fitting of a model, it becomes necessary to verify its future performance, i.e. to measure the generalization capability in unseen data. We adopted the Leave-One-Out scheme for such purpose. This method is especially suited when the dataset is small (e.g. lower than 100 examples). Under Leave-One-Out, sequentially one example is used to test the model and the remaining data is used for fitting the model. Under this scheme, all data is used for training and testing. Yet, this method requires around $\mathrm{N}$ (the number of data samples) times more computation, since $\mathrm{N}$ models are fitted. The final generalization estimate is evaluated by computing the MAD, RMSE and $\mathrm{R}^{2}$ metrics for all $\mathrm{N}$ test samples.

To understand better the behaviour of the JG material, the influence of each parameter was quantified. To do this, we applied sensitivity analysis procedure [20][17]. This process determines the most important variables in a system by successively holding all but one input constant and varying the other over its range of values to observe its effect on the system. A high variance observed in the outputs denotes a high input relevance.

\section{RESULTS}

The quality of the predictions obtained by applying the EC2 analytical model confirms the good performance of the predictions, presenting small values of MAD $=0.60$ and
$\mathrm{RMSE}=0.88$ and a correlation closely to unit $\left(\mathrm{R}^{2}=0.91\right.$, Fig. 3). However, despite its good accuracy, to apply successful EC2 analytical model it is necessary to know the UCS of each formulation over the 28 day time-frame of the cure, which implies waiting and carry out laboratory tests to determine the UCS at this age. Furthermore, an inadequate value of $f_{c m}$ leads to significant errors in the predictions.

A high performance was obtained for both ANN and SVM. Despite ANN presented the best performance (Fig. 3), it was not considered further since the importance attributed by this model to each parameter is not in agreement with the empirical knowledge of JG technology (Fig. 4). According to SVM, we can say that the key parameters to predict the UCS of JG over time are: the time of cure, the percentage of cement of the mixture and the relation $n /\left(C_{I V}\right)^{d}$. It can also be verified that the properties of soil, rather the amount of organic matter and silt, are also relevant to evaluate the behavior of the soil cement mixtures over time. When comparing SVM and EC2, the former is better, as shown in Fig. 3. In contrast with EC2, the SVM allows prediction of the UCS in the initial stages of a project. However, despite this advantage and its high predictive accuracy, the SVM model is very complex and difficult to understand by humans.

After we train the FN, from (5), using the Leave-On-Out estimation method and the minimization problem (6), we obtain the performance shown in Fig. 2, which represents the relation between the measures and predicted Jet Grouting UCS values, given by the expression:

$$
\begin{aligned}
q= & 12023149 \cdot\left(W / C^{-1.052} \cdot s^{-2.090} \cdot t^{0.239}\right) \cdot \\
& \cdot\left(n /\left(C_{I V}\right)^{0.04}\right)^{-3.064} \cdot \% C^{1.473} \cdot \% \text { Sand }^{0.028} \\
& \cdot \% \text { Silt }^{-1.594} \cdot \% \text { Clay }^{0.397} \cdot \text { OM }^{-0.295}
\end{aligned}
$$

The high performance obtained is corroborated by the MAD, RMSE and $\mathrm{R}^{2}$ error metrics (see Fig. 3). The values of the two first are $0.58 \mathrm{MPa}$ and $0,75 \mathrm{MPa}$ respectively, which are small values and the correlation presents a value of 0.92 , which is very close to the unit value (the perfect prediction). Moreover, as can be seen in Fig. 2, the number of predictions above of ideal prediction (black line in Fig. 2) is approximately equal to number of all predictions below the same line, revealing that the model does not underestimate or overestimates the predictions. The importance of each FN parameter was also computed using a sensitivity analysis procedure [20]. Similar to the SVM model, the amount of cement and the relation $\mathrm{n} /\left(\mathrm{C}_{\mathrm{iv}}\right)^{\mathrm{d}}$ are the most important parameters in prediction of the UCS JG laboratory formulation. It is still possible observe that the properties of soil, rather the amount of organic matter and silt, are also relevant (as in SVM model).

When compared to SVM, the FN model is more interesting since its mathematical expression is simple and understandable, while the obtained predictive accuracy is still high. In addition, we can yet apply FN model in the initial stage of a project, what is impossible with EC2 analytical model. Table III summarizes the advantages and disadvantages of the proposed approach. 
TABLE III. COMPARISSON OF THE PREDICTIVE MOdELS

\begin{tabular}{cccc}
\hline Model & $\mathbf{R}^{2}$ & $\begin{array}{c}\text { Applied in } \\
\text { project stage }\end{array}$ & $\begin{array}{c}\text { Easy to interpret } \\
\text { by humans }\end{array}$ \\
\hline EC2 & 0.91 & $\times$ & $\checkmark$ \\
ANN & 0.94 & $\checkmark$ & $\times$ \\
SVM & 0.93 & $\checkmark$ & $\times$ \\
FN & 0.92 & $\checkmark$ & $\checkmark$ \\
\hline
\end{tabular}

\section{CONCLUSIONS}

The Jet Grouting (JG) is one of the most popular ground improvement techniques. However, the main JG works are planned from empirical rules that are often too conservative. In this work, we propose a novel data-driven approach to predict the Uniaxial Compressive Strength (UCS) of JG laboratory formulations over time.

A comparison among the four models tested reveals the Functional Networks (FN) has the best choice (Table III). This model presents a good performance and it is understandable by humans. Also, it can be applied during the initial stage of a project, in opposition to EC2. The key parameters that control the behaviour of JG laboratory formulations were also identified: the amount of cement and the relation $n /\left(\mathrm{C}_{\mathrm{iv}}\right)^{\mathrm{d}}$ and the properties of soil. It should be stressed that tested models are only valid for the conditions found in the dataset used in this study (e.g., clay soil type).

The best solution, the FN model, can give a valuable contribution in terms of improving the construction process of JG columns and reducing the costs of laboratory formulations. In face of the results obtained, we intent apply similar Data Mining (DM) techniques, to predict the Young modulus of JG laboratory formulations, as well as to estimate the final diameter of JG columns and its mechanical properties (UCS and Young modulus).

\section{ACKNOWLEDGMENTS}

The authors would like to thank the interest and financial support by Tecnasol-FGE and Tiago Valente for the dataset gathered from the laboratory formulations.

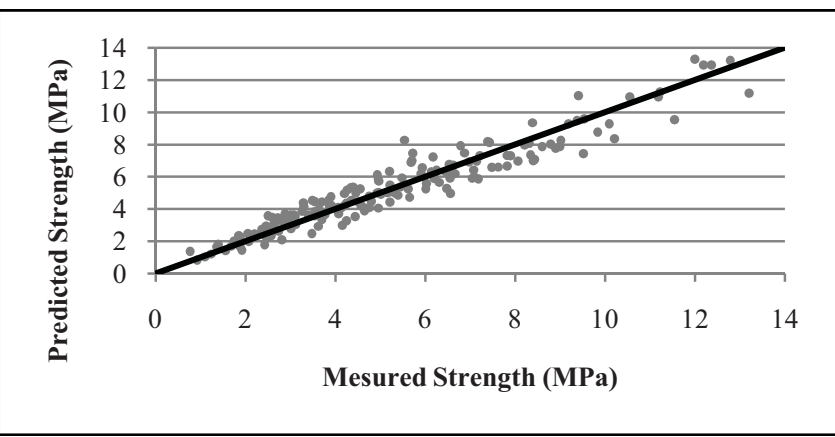

Figure 2. Predicted versus measured strength of JG laboratory formulations using the FN model

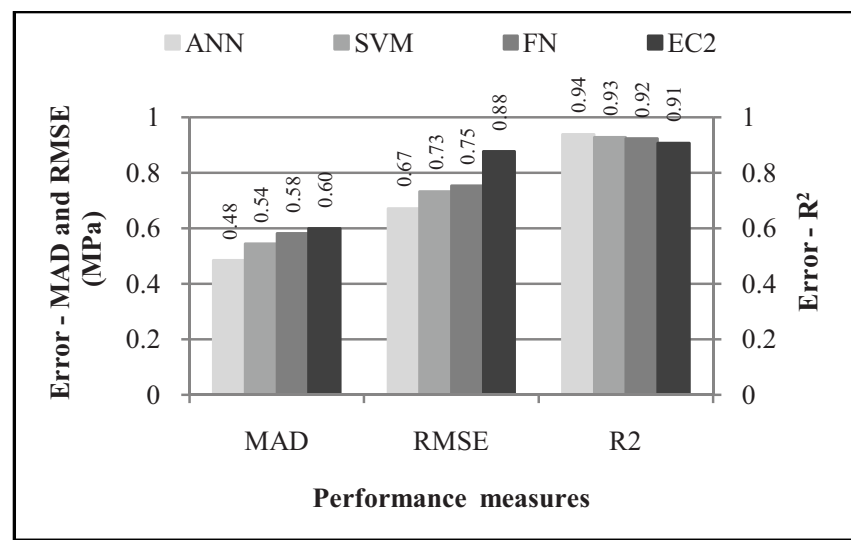

Figure 3. Comparison of the performance between the four models: EC2, SVM, ANN and FN

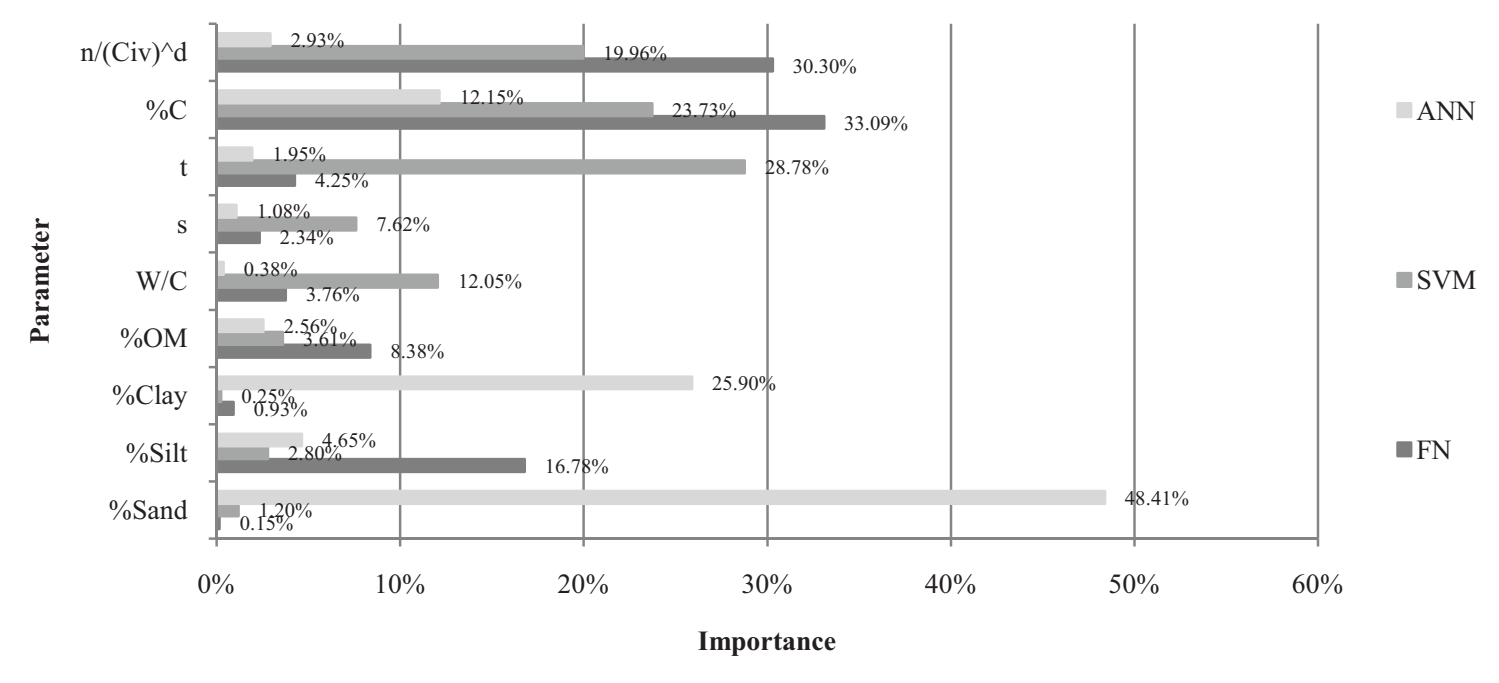

Figure 4. Comparison of the importance of each parameter in the four models: EC2 analytical model, SVM, ANN and FN models 


\section{REFERENCES}

[1] GmbH, Keller Holding. "The soilcret - Jet Grouting Process.” Keller Publications. 2005.

[2] Falcão, João F., Alexandre L. Pinto, and Francisco D. Pinto. "Cases histories of ground improvement solutions using jet-grouting." 2000.

[3] Padura, A. Barrios, J. Barrios Sevilla, and J. García Navarro. "Study of the soil consolidation using reinforced jet grouting by geophysical and geotechnical techniques: "La Normal" building complex (Granada)." Construction and Building Materials (2008), doi:10.1016/j.conbuildmat.2008.07.011

[4] Croce, P., and A. Flora. "Analysis of single-fluid jet grouting." Géotechnique ,Vol. 51 (2001), pp. 905 - 906.

[5] Kirsh, Fabian, and wolfgang Sondermann. "Ground Improvement and its Numerical Analysis.” Istanbul, 2001.

[6] Normalisation, Comité Européen the. Eurocode 2: "Design of concret structures - Part 1-1: General rules and rules for buildings." Bruxelas: CEN, 2004.

[7] D. Rumelhart, G. Hinton, and R. Williams. "Learning Internal Representations by Error Propagation." In D. Rulmelhart and J. McClelland, editors, Parallel Distributed Processing: Explorations in the Microstructures of Cognition, vol. 1, pp.318-362, MIT Press, Cambridge MA, 1986.

[8] C. Cortes and V. Vapnik. "Support Vector Networks." Machine Learning, 20(3):273-297, 1995.

[9] T. Hastie, R. Tibshirani, and J. Friedman. "The Elements of Statistical Learning: Data Mining, Inference, and Prediction.” Springer-Verlag, NY, USA, 2001.

[10] Castillo, Enrique, José Manuel Gutiérrez, Ali S. Hadi, and Beatriz Lacruz. "Some Applications of Functional Networks in Statistics and Engineering." Technometrics 43 (2001), pp.10-24.

[11] El-Sebakhy, Emad A. "Software reliability identification using functional networks: A comparative study." Expert systems with applications 35 (2009), pp. 4013-4020.

[12] Castillo, Enrique, Ali S. Hadi, Beatriz Lacruz, and Rosa E. Pruneda. "Semi-parametric nonlinear regression and transformation using functional network." Computational Statistics \& Data Analysis, 2008, pp. 2129-2157.

[13] Betanzos, Amparo Alonso, Enrique Castillo, Fontenla Oscar Romero, and Noelia Sánchez Marono. "Shear Strength Prediction using Dimensional Analysis and Functional Networks." European Symposium on Artificial Neural Networks. Bruges (Belgium), 2004, pp.251-256.

[14] Rezania, Mohammad, and Akbar A. Javadi. "A new genetic programming model for predicting settlement of shallow foundations." Canadian Geotechnical, December 2007, pp.14621473.

[15] Yusuf E. "Artificial Neural Networks Approach for Swell Pressure versus Soil Suction Behavior." Canadian Geotechnical, 2007, pp.1215-1223.
[16] Lai S., Serra M. "Concrete Strength Prediction by means of Neural Network." Constrruct Build Mater (1997); 11(2), pp. 93-98.

[17] Shibazaki, Mitsuhiro. "State of Pratice of Jet Grouting." Grouting and Ground Treatment. New Orleans (2003), pp.198-217.

[18] Gazzarrini, Paolo, Matt Kokan, e Stephen Jungaro. "Case History of Jet Grouting in British Columbia. Underpinning of CN Rail Tunnel in North Vancouver." The Grout Line (2005), pp. 47-54.

[19] Jalali, Saíd, e L.M.V. Santos. "Early Quality Control and Early Formwork Removal whith setting Time." 1996.

[20] Kewley, R., Embrechts, M and Breneman, C. "Data Strip Mining for the Virtual Design of Pharmaceuticals with Neural Networks". IEEE Transactions on Neural Networks, 11(3), 2000, pp.668-679.

[21] Kenig, S., Ben-David, A., Orner, M., and Sadeh, A. 2001. "Control of properties in injection molding by neural networks." Engineering Applications of Artificial Intelligence, 14, pp.819-823. doi: J O. 1016IS0952-1976(02)00006-4.

[22] Perzyk, M., and Kochanski, A.W. 2001. "Prediction of ductile cast iron quality by artificial neural Networks." Journal of Materials Processing Technology, 109, pp.305-307. doi: 10. 10 16/S09240136(00)00822-0.

[23] Smola A. and Scholkopf B. "A tutorial on support vector regression." Statistics and Computing (2004), pp.14:199-222.

[24] V. Cherkassy and Y. Ma. "Practical Selection of SVM Parameters and Noise Estimation for SVM Regression." Neural Networks, 17(1), pp. 113-126, 2004.

[25] Castillo, E., A. Cobo, J. M. Gutiérrez, and E. Pruneda. "Functional Networks with Applications: a neural-based paradigm." Springer, 1998.

[26] El-Sebakhy, Emad A., Kanaan Abed Faisal, T. Helmy, F. Azzedin, and A. Al-Suhaim. "Evaluation of breast cancer tumor classification with unconstrained functional networks classifier." In Proceeding of the 4th ACS/IEEE international conference on computer. (2006), pp.281-287.

[27] Li, Chunguang, Xiaofeng Liao, Zhongfu Wu, and Juebang Yu. "Complex functional networks." Mathematics and Computers in Simulation (2001), pp.355-265.

[28] Zhou, Yong Quan, Deng Xu He, and Zheng Nong. "Problems, Application of Functional Network to Solving Classification." World Academy of Science, Engineering and Technology. 2005. 390-393.

[29] R Development Core Team. "R: A language and environment for statistical computing." R Foundation for Statistical Computing, Vienna, Austria, ISBN 3-900051-00-3, http://www.R-project.org, (Accessed July 27, 2009).

[30] Cortez, Paulo. "RMiner: Data Mining with Neural Networks and Support Vector Machines using R.” In R. Ra jesh (Ed.), Introduction to Advanced Scientific Softwares and Toolboxes, In press.

[31] GAMS, Development Corporation. "On-line Documentation." Welcome to the GAMS Home Page. Juny 15, 2009. http://www.gams.com/docs/document.htm (accessed Juny 27, 2009) 